\title{
GATOPARDISMO Y ENSEÑANZA DE LA HISTORIA. ANÁLISIS DE LOS PLANES Y PROGRAMAS DE ESTUDIO PROPUESTO POR EL NUEVO MODELO EDUCATIVO PARA LA EDUCACIÓN BÁSICA EN MÉXICO
}

\author{
Everything to change so everything stays the same in teaching history. Analysis of \\ the plans and study programs proposed by the New Educational Model for basic \\ education in Mexico
}

\section{Xavier Rodríguez Ledesma \\ Sebastián Plá}

Fecha de recepción: 03/07/2018

Fecha de aceptación: 29/12/2018

RESUMEN: México ha vivido en reforma educativa permanente desde los años setenta del siglo XX a la fecha. El ascenso al poder del nuevo presidente mexicano Andrés Manuel López Obrador parece no modificar esta tendencia, especialmente en lo que la enseñanza de la historia se refiere. Ante los nuevos cambios por venir, es pertinente preguntarse ¿cuáles han sido las modificaciones más significativas en la enseñanza de la historia en la última reforma educativa mexicana? ¿Qué permanece y qué cambia del código disciplinar? ¿Cómo pensar una enseñanza de la historia más inclusiva para el futuro? Para responder a estas preguntas, analizamos aquí el programa de estudio para la asignatura de historia de Nuevo Modelo Educativo publicado en 2017. El análisis asume tres niveles reflexivos: a) el estudio del currículo, b) los saberes o disciplinas escolares como productos históricos, y, c) la pedagogía por la justicia social. Desde la perspectiva construida por estos tres ejes el texto encuentra que, a pesar de los esfuerzos por reformar los enfoques de los procesos de enseñanza aprendizaje de la historia en la educación básica, la propuesta construida hace suya una serie de puntos epistemológicos, historiográficos y políticos que ha caracterizado tradicionalmente a los programas de historia en México: la importancia de la enseñanza de las habilidades heurísticas del historiador, la permanencia del Estado-nación mexicano como sujeto primordial de la historia y la permanencia de la identidad única, uniforme y excluyente del mestizo mexicano.

PALABRAS CLAVE: Aprendizaje, Historia, Educación básica, Reforma educativa, México.

ABSTRACT: Mexico has been living in a continuous educational reform since the seventies of the last century. The election of a new president, Andres Manuel López Obrador, in 2018 do not seems it will change this historical tendency. Before the changes for become, we ask, what were the most meaningful changes for History Education in the last educational reform? What changed and what remain? How can we think a more inclusive history teaching for the future? To answer these questions, we will analyze the new syllabus for teaching and learning History for compulsory education in Mexico, prescribed in the "New Educational Model" and published in 2017 by Enrique Peña Nieto (2012-2018) administration. The analysis assumes three reflective levels: a) the study of the curriculum, b) school knowledge about history as a historical product, and c) pedagogy for social justice. From the perspective constructed by these three axes, the text finds that, despite the effort to renewed the teaching history perspective for primary and secondary 
education, the new syllabus endorses a series of epistemological, historiographic and political points that has traditionally characterized the history programs in Mexico: the historian skills like only legitimized path to known the past, the Nation-State as core subject of the current of history and the homogeneous - and discriminatory- Mexican-mestizo identity. Finally, we do some general comments about the urgent need for history programs for basic education to assume the epistemological, historiographic and political consequences that cultural diversity, finally recognized, implies to promote a social justice pedagogy.

KEY WORDS: History Education, Compulsory education, Mexico, Educational reform, Curriculum.

\section{Introducción. El Nuevo Modelo Educativo 2017 para la educación básica en México}

Desde la entrada del neoliberalismo a la mexicana en los años ochenta del siglo XX con los gobiernos del Partido Revolucionario Institucional (PRI), es decir, con la implementación de políticas desreguladoras, de privatización de las paraestatales y de la reducción del gasto social basado en el férreo control clientelar y sindical de los diferentes ramos (Meyer, 1995) la educación mexicana ha vivido en una reforma permanente. Dicha reforma permanente inició a principios de los años setenta del siglo pasado, con los procesos de tecnología educativa, la pedagogía por objetivos (Latapí, 1998) y la noción de aprender a aprender (Plá, 2018). A finales de esa misma década y durante los años ochenta, se inició con una tímida federalización del sistema educativo (Ornelas, 2000) y un violento embate contra el salario y el prestigio de la profesión docente (Arnaut, 1998). En la última década del siglo XX la reforma en México y en el resto de América Latina, se profundizó su acción en tres ejes: nuevas formas de gestión del sistema educativo, la profesionalización docente y el diseño curricular (Tedesco, 2016).

Con el fin de setenta años de gobierno del Partido Revolucionario Institucional (PRI) con el triunfo electoral de Vicente Fox Quesada del Partido Acción Nacional (PAN) en el año 2000, la reforma educativa permanente no se detuvo sino, por el contrario se aceleró. En 2002, se declaró obligatoria la educación preescolar, en 2006 el Diario Oficial de la Federación publicó los nuevos programas para la educación secundaria y en 2009 se inició el proceso denominado Reforma Integral a la Educación Básica en México (RIEB), que concluyó en 2011 con nuevos enfoques y contenidos para preescolar, primaria y secundaria. De igual forma en ese año se realizó la reforma a la licenciatura en educación primaria de las escuelas normales y un año después se declaró el nivel de bachillerato educación obligatoria, como resultado de la Reforma Integral a la Educación Media Superior (RIEMS) iniciada en 2008. También durante el panismo se fundó el Instituto Nacional de Evaluación Educativa en 2002, dispositivo central para la gobernación del sistema educativo mexicano (Aboites, 2012). El resultado es un sistema educativo cuya columna vertebral está compuesta por el preescolar, la primaria y la secundaria que juntos conforman la educación básica y obligatoria, y a lo que se le suma el bachillerato, que es educación obligatoria pero no básica. Todo lo anterior se desarrolló con base en el neoliberalismo a la mexicana, es decir, con base en el control del sindicalismo magisterial (Ornelas, 2000, 2012; Alcántara, 2008; Plá, 2018).

Con la recuperación de la presidencia por parte del PRI bajo el liderazgo de Enrique Peña Nieto en 2012, la reforma educativa no sólo continuó, sino que se convirtió en la punta de lanza de nuevas reformas estructurales (energética y comunicaciones entre otras) impulsadas por el nuevo 
gobierno. En unos cuantos meses, bajo el auspicio del partido oficialista y la mayoría de los partidos de oposición, se modificó la Constitución Política declarando a la calidad educativa como un derecho, al mismo tiempo que se establecía un régimen laboral de excepción para los docentes. Además, se modificó la Ley General de Educación y se crearon dos leyes secundarias más: la Ley de Instituto Nacional de Evaluación Educativa, que daba autonomía al instituto y le imponía nuevas obligaciones (evaluar y certificar docentes), y la Ley del Servicio Profesional Docentes, donde se puntualizaba el control sobre el ingreso y la permanencia de los docentes. Estas leyes, en especial la última, generaron fuertes movilizaciones de la disidencia magisterial encabezada por la Coordinadora Nacional de los Trabajadores de la Educación (CNTE). Los primeros tres años de reforma se centraron en el esfuerzo gubernamental por mitigar la oposición y establecer el nuevo sistema de evaluación docente. Esto hizo que la policía federal y sus toletes se convirtieran en actores directos de la reforma educativa.

Las críticas a la reforma se pueden sintetizar en una frase muy repetida: "Es una reforma mal llamada educativa, es una reforma laboral" (CNTE, 2013), pues parte nodal de la reforma se concentró en el ingreso y permanencia de los docentes. Dicha crítica tenía fundamentos, pero era claramente insuficiente. La reforma, en efecto, tocaba puntos laborales pero no debemos olvidar que la condición laboral también modifica la vida en la escuela. Por su parte, desde la investigación educativa la crítica se ha referido al establecimiento de un sistema de evaluación docente sin que se hubiera definido en primer lugar qué, para qué y cómo se pretende educar en la escuela (Díaz Barriga, 2016; Gil Antón, 2013). En otras palabras, las cosas se habían hecho al revés ya que en vez de diseñar en primer lugar un nuevo modelo educativo para después establecer sus mecanismos de evaluación y medir sus alcances y logros, se estableció un sistema de evaluación en aras de consolidar un control laboral como valor en sí mismo (Rockwell, 2015).

Para responder a dichas críticas en 2016 se publicó el Nuevo Modelo Educativo cuya versión definitiva apareció en 2017. Dicho modelo tiene como objetivo principal dar fundamento pedagógico al sistema de evaluación previamente establecido. Este orden de los acontecimientos no es menor, pues epistemológicamente, el conocimiento curricular que se legitima y se prescribe, ha de ser diseñado con una condición básica: debe poder evaluarse (Rockwell, 2015, Plá, 2018). Lo que no pueda medirse - evaluarse- queda fuera de dicho modelo. Fijados sus límites, el modelo educativo 2017 tiene cinco dimensiones. La primera es la escuela al centro, que promueve nuevas formas de gestión; la segunda es la formación de los docentes; la tercera la inclusión y la equidad como principios rectores, la cuarta la gobernanza del sistema, donde se ubica el Instituto Nacional de Evaluación Educativa (INEE) y da entrada a la participación privada a la educación; y el quinto el nuevo planteamiento curricular (SEP, 2017a). Sobre este último punto, que es el objeto de análisis del presente texto, es necesario ahondar en sus dimensiones históricas, epistemológicas y políticas que lo componen.

Si leemos el NME desde su dimensión histórica, se pueden distinguir procedencias de muy diversa índole. Por un lado, recupera los principios neurocientíficos del aprendizaje impulsados por la OCDE en The Nature of Learning (2012), como son el papel de las emociones en el desarrollo de los aprendizajes y lo que se denomina aprendizaje-servicio, es decir, una afirmación del conocimiento social basado en principios de beneficencia. El Nuevo Modelo abreva del proceso de tecnología educativa de los años setenta en México, proceso curricular interesante que imbricó el eficientismo curricular tyleriano y en especial la pedagogía por objetivos de Bloom (Latapí, 1998), las técnicas Freinet, consolidadas en México tras treinta años de exilio republicano español 
en el país y un dejo de principios marxistas dentro de un Estado autoritario y centralista. Aprender a aprender es el concepto que condensa los elementos anteriores, concepto que se comenzó a usar en México simultánea e incluso anteriormente que la publicación del informe Fauré de 1974 (Plá, 2018).

Hoy, el Nuevo Modelo recupera la idea de aprender a aprender, conserva el eficientismo curricular a través de aprendizajes esperados susceptibles de ser evaluados, mantiene algunas técnicas Freinet -sobre todo en las emociones y el niño al centro-, elimina todo vestigio marxista y de conocimiento crítico de la realidad social y conserva el centralismo de manera particular: ofrece autonomía curricular al centro escolar (SEP, 2017a), después de establecer un mecanismo de vigilancia que mide que tan autónomo eres, a imagen y semejanza de lo que ha sucedido en Estados Unidos (Apple, 2012). La historia conceptual del NME educativo es lo que hemos denominado saqueo de adjetivos, es decir, el proceso mediante el cual el capitalismo contemporáneo logra sumar a su discurso las demandas de sus críticos sin modificar la estructura de desigualdad que lo constituye (Plá, 2018). De ahí los resultados cuasi esquizoides, donde se promueven técnicas Freinet en contextos de evaluación intensivos, masivos y punitivos.

En el aspecto epistemológico, el NME educativo imbrica la larga tradición del código disciplinar de la historia que hace de ésta en la escuela algo diferente a la historiografía profesional (Chervel 1991, Cuesta 1998, Chevalard, 1997). El resultado sea una amalgama entre nacionalismo y método del historiador, desgranado este último en habilidades cognitivas (Plá, 2014).

Por último, la lectura de la dimensión política del currículo, enfocada desde la pedagogía de la justicia social, muestra cómo la historia de las reformas educativas de los últimos treinta años y su posición epistemológica, produce nuevas formas de exclusión simbólica que reproducen las condiciones estructurales de desigualdad. Básicamente, esta se da en tres ámbitos: la identidad homogénea, la relación jerárquica entre saberes y el tipo de conocimiento social que se favorece. La primera, que es la de más larga data, consiste en reproducir un metarrelato nacional que naturaliza un discurso de mediados del siglo XX en el cual se sostiene que lo mexicano es lo mestizo, nacido del sincretismo entre lo español y lo mestizo (Plá, 2014a; Casal, 2011, Young, 2010). A esta visión le subyace un profundo racismo que niega, a su vez, el reconocimiento constitucional de México como país pluricultural de 2002. Los afrodescendientes, la pluralidad de los grupos indígenas, las migraciones de los siglos XIX y XX, se omiten de las nuevas narraciones curriculares, a pesar de que, paradójicamente, los programas para educación secundaria del gobierno conservador de 2006, tendían a valorarlo más (Ballesteros 2015, Rodríguez Ledesma 2014). El segundo aspecto es la relación jerárquica entre saberes, aspecto que las teorías de la decolonialidad han denunciado para todo el continente (Walsh 2006, Mignolo 2003, Quijano 2000). En el caso de la enseñanza de la historia, este proceso se centra en la cada vez mayor centralidad del método del historiador concebido como conjunto de habilidades cognitivas universales que permiten un acercamiento verdadero al pasado. Además, se tiende a eliminar la historia indígena (Pacheco, Navarro y Cayeros, 2011). No negamos el valor del conocimiento producido por los historiadores profesionales, sino que lo que denunciamos es cómo una forma occidental de pensar se impone como universal, limitando las posibilidades de trabajar en las aulas múltiples maneras legítimas y válidas de narrar el pasado. Así, por ejemplo, la tradición oral de las comunidades es vista como conocimiento inferior que no tiene suficiente legitimidad científica para convertirse en conocimiento escolar. Esto, evidentemente, reproduce la desigualdad simbólica. 
El tercer elemento de la dimensión política tiene que ver con la formación ciudadana. En el NME y en sus manifestaciones disciplinares, en especial en las asignaturas de Historia y de Formación Cívica y Ética, hay un vaciamiento muy notable de los contenidos socialmente relevantes (Santisteban 2010, Legardez 2003, Pérez Exposito, 2014), específicamente de todos aquellos relacionados con las condiciones de desigualdad producidas por el capitalismo contemporáneo. Como veremos más adelante, los contenidos relacionados con formas de dominación, pobreza, racismo y derechos sociales se han desvanecido reforma tras reforma curricular y son sustituidos por lo que denominan, arrastrados por la fuerza de la OCDE, voluntad de servicio o aprendizaje de servicio -en el modelo se menciona como servicio comunitario (Dumont et al., 2010)-. Es decir, una solidaridad voluntarista, no estructural, una participación ciudadana basada en la beneficencia y no en la lucha por la justa redistribución de la riqueza por medio de los derechos sociales, el reconocimiento a la diversidad y la adecuada representación política. De esta manera, el NME, en su dimensión política, es incapaz de garantizar a través de la escuela, entre muchas otras cosas, el derecho de los pobres a saberse en cuanto tales (González Arroyo, 2015). Este aspecto, central en cualquier pedagogía que promueva la justicia social, es uno de los grandes olvidos de la nueva prescripción curricular mexicana.

\section{Apuntes teórico-metodológicos}

Este artículo es un esfuerzo por hacer un análisis curricular, desde la pedagogía por la justicia social, de los planes y programas de estudio de historia propuestos por el Nuevo Modelo Educativo (NME) dados a conocer en México durante el año 2017. Nuestro acercamiento crítico abreva teórica y metodológicamente de tres fuentes: a) los estudios del currículo, que conciben a éste como un discurso político, histórico y cultural (Pinar, 2004), b) la concepción de saber escolar y de la cultura escolar, y, c) las pedagogías por la justicia social que tienen la finalidad de deconstruir los marcos simbólicos de dominación.

Si bien los estudios del currículo se crearon hace más de cien años fue en la década de los setenta del siglo pasado cuando se perfilaron tres ejes analíticos fundamentales: a) recuperar el papel de la clase social y la legitimación del saber prescrito en el currículo, b) plantear una oposición frontal al eficientismo tyleriano y a la racionalidad conductista tanto del currículo como de las prácticas escolares, y c) reconocer de forma explícita el conflicto -y las relaciones de poder que le subyacen- en el diseño curricular. En los últimos lustros se han agregado a este campo de análisis múltiples lecturas del currículo desde diferentes perspectivas, como las históricas, las políticas, de género, las fenomenológicas (García Garduño, 2014) y, en los últimos tiempos, desde la justicia social (Chappman y Hobbel, 2010; Plá, 2016). En el presente trabajo nos limitamos a las dimensiones histórica, epistemológica y política del currículo en su manifestación programática, es decir, en el diseño curricular.

Por lo que respecta a las disciplinas o saberes escolares, se conciben aquí como producto histórico que se consolida dentro de la escuela como resultados de luchas de poder entre grupos universitarios o políticos que combaten por la legitimación social de su saber. La aparición de la historia o las matemáticas como contenido escolar es consecuencia en primera instancia de las funciones sociales que se le otorgan a dicho conocimiento, pero sobre todo al triunfo de determinados grupos de poder-saber (Goodson,1995). Este análisis, al igual que la producción social del conocimiento escolar de Popkewitz, muestra cómo el valor de un saber escolar no está dado por sus elementos intrínsecos, sino por relaciones de poder por fuera de la escuela. sin 
embargo, dentro de la escuela, dichos saberes se metamorfosean y se piensan, diseñan, planifican y producen bajo criterios escolares y no científicos. (Chervel 1991; Viñao 2006). Ahora bien, a pesar de que la cultura escolar imprime elementos comunes a cada saber escolar, estos poseen códigos disciplinares específicos, es decir, un conjunto de normas y prácticas escolares que le dan sentido dentro de la escuela (Cuesta 1998; Plá 2012). Así, la historia de la enseñanza de la historia en el currículum es la historia de un saber escolar, no la historiografía de un saber científico.

La tercera fuente de la que abreva nuestro análisis es lo que se ha denominado, de manera genérica, la pedagogía por la justicia social. Dicha pedagogía, al igual que la reconceptualización del currículo, conjuga diversos enfoques teóricos, por lo que retoma del posestructuralismo, los estudios culturales, la decolonialidad, los estudios poscoloniales y la pedagogía crítica, emergida desde la nueva sociología de la educación y de la reconceptualización del currículo. Los cuatro elementos básicos de dicha pedagogía son: a) desafiar, confrontar y producir rupturas en los estereotipos, las falsedades y los conceptos erróneos que guían las desigualdades y las discriminaciones estructurales basadas en clase social, género, fenotipo, cultura y otras diferencias entre los seres humanos; b) proveer los elementos educativos necesarios para que todos los estudiantes puedan desarrollar su potencial; c) aprovechar las fortalezas de los estudiantes en su propia educación; y d) crear ambientes de aprendizaje que promuevan el pensamiento crítico como agente del cambio social (Chapman and Hobbel, 2010:1-2). En el presente trabajo nos centraremos en el primer punto arriba señalado.

Con base en lo hasta aquí expuesto podemos decir que nuestra teoría abreva de la reconceptualización del currículo, los saberes escolares y la pedagogía por la justicia social y que la metodología es cualitativa. Esto implica entender el currículo no como un plan de estudios -y sus programas por asignatura- estable y fijo, que se opera de igual manera en todas las escuelas, sino que se reconfigura por lo menos en dos planos: la experiencia en el aula y la planificación escolar. En este momento, nos interesa el currículo en su plano programático. Dicho plano es un discurso histórico, político y epistemológico que produce un saber que responde más a los procesos de escolarización, incluso con su propia epistemología, que imbrica diferentes posicionamientos que tienden a reproducir las condiciones simbólicas que subyacen en la desigualdad estructural de nuestra sociedad. La pedagogía por la justicia social nos obliga a fijar nuestra atención en dichos aspectos y buscar otros caminos para la emancipación. Metodológicamente, partimos de lógicas interpretativas que se basan en lectura a profundidad y que, sin demeritar la metodología de análisis formal del discurso, creemos que son más útiles para comprender el sentido de la narración histórica curricular.

Nos interesan las dimensiones históricas, epistemológicas y políticas de los nuevos planes de estudio para la enseñanza de la historia y no los aspectos técnicos del diseño curricular (Abellán, 2018), por lo que seleccionamos tres objetos de análisis: la relación entre el código disciplinar de la historia frente a visiones alternativas de la historia escolar (dimensión histórica); la relación entre el pasado como saber social frente a la expansión del pensamiento histórico como imposición cognitiva (dimensión epistemológica) y las concepciones de política, ciudadanía y sujetos de la historia (dimensión política). Estas tres dimensiones se conjugan en la perspectiva de la justicia social, es decir, analizar qué tanto los contenidos históricos logran romper con los estereotipos de dominación de clase, género y raza y dan sustento simbólico a la desigualdad en México. Para enmarcar esto, es necesario exponer, aunque sea sucintamente, la reforma educativa y los principios 
que guían el Nuevo Modelo Educativo y las dimensiones históricas, políticas y epistemológicas que le subyacen.

Para nuestro análisis nos basamos en dos fuentes. La primera es el documento de presentación del NME (SEP, 2017 $)$. La segunda son los planes y programas de estudio del NME que se encuentran en: http://www.aprendizajesclave.sep.gob.mx/ de la cual existe una versión impresa de dicho documento (SEP 2017b) que no reproduce la totalidad de los contenidos que aparecen en la página de internet ${ }^{1}$.

\section{Resultados}

\section{La dimensión politica}

En la presentación general del NME se expresa con claridad el posicionamiento político que lo inspira. Ahí el entonces secretario de educación pública Aurelio Nuño, escribió que el objetivo de la "auténtica revolución educativa" impulsada por su propuesta era "garantizar el acceso a la educación, asegurar que esta (sic) sea de calidad y se convierta en una plataforma para que los niños, niñas y jóvenes de México triunfen en el siglo XXI. Debemos educar para la libertad y la creatividad" (SEP, 2017a: 7. Negritas nuestras). Más allá de la ironía consistente en la falta de ortografía que ahí aparece, el verbo elegido para sintetizar la característica fundamental del perfil de egreso contemplado por el NME es muy puntual: "triunfar". De ahí que sea evidente que actualmente el objetivo de la educación básica en nuestro país no sea formar niños, niñas y jóvenes más solidarios, empáticos, colaborativos, conscientes del poder intrínseco en el accionar comunitario o algún otro tipo de valor colectivo. No, nada de eso, se trata de hacerlos "triunfadores", ganadores individuales por sobre el resto de la comunidad conformada por, obvio, perdedores. La cultura emprendedora del capitalismo actual, quedó plasmada desde los renglones iniciales del documento.

Respecto a los planes y programas de historia saltan a la vista dos particularidades que los definen. La primera característica es su talante político e ideológico, pues al asumir como objetivo central el añejo anhelo de construir identidad nacional, se generan a su vez dos consecuencias de índole política:

a) se deja de lado la posibilidad de que la enseñanza de la historia tuviera como fin identificar a otras utilidades y funciones sociales que podrían apuntar a la construcción de una sociedad signada por el reconocimiento de las múltiples diversidades que conforman a realidad política, cultural y social contemporánea, y,

b) se ubica al Estado nación como eje alrededor del cual girarán todos los contenidos temáticos de los programas (hechos, periodizaciones, coyunturas, protagonistas, etc.)

La segunda característica general refiere al carácter epistemológico de la historia pues los programas parten de la convicción de que sólo existe una forma de acceder y legitimar dicho conocimiento: la ciencia histórica. Esta posición constituye una contradicción epistémica (y política) pues significa que el pensamiento crítico debe detener sus afanes problematizadores, cuestionadores y analíticos en cuanto el templo de la cientificidad aparece en el horizonte discursivo.

\footnotetext{
${ }^{1}$ Nos referimos a las "Orientaciones didácticas" y a las "Sugerencias de evaluación" elaboradas para cada uno de los ejes temáticos de los diversos programas por grado.
} 
Regresemos a lo señalado en el documento. El breve apartado que define la importancia de la historia en la educación básica concluye así: “... la historia en educación básica es una fuente de conocimiento y de formación para que los alumnos aprendan a aprender y a convivir con los saberes que proporciona a partir del pensamiento crítico, el análisis de fuentes y de promover valores que fortalezcan su identidad.”, (SEP 2017b, 383)

Un análisis cuidadoso de éstas líneas arroja tres puntos nodales:

1. “Aprender a aprender". La frase hace suya una idea pedagógica de larga data, a pesar de presentarse como innovadora. Dicha propuesta debería ser capaz de responder de forma concreta preguntas como las siguientes: ¿qué contenidos son los que los alumnos deben aprender a aprender?, es decir, ¿qué tipo de historia los estudiantes deben aprender a aprender? Ambas preguntas son importantes puesto que el objetivo de la educación no puede definirse por una tautología, sino que es necesario concretarlo en una definición puntual de los saberes que habrán de construirse a través del proceso de aprendizaje. Para el documento analizado la respuesta es simple: el estudiante debe aprender a aprender la forma en que la historia hegemónica, legitimada y constituida como disciplina escolar plantea el devenir de la humanidad.

2. "Convivir con saberes que la historia proporciona a través del pensamiento crítico". ¿Qué quiere decir para la SEP tan sugerente frase? No lo sabemos. La redacción es poco clara y la información ofrecida por el NME no ahonda más allá de entender el pensamiento crítico como un conjunto de habilidades para el análisis de fuentes. Pareciera que la intención fue usar a como diera lugar el concepto "convivir" ya que éste refiere a una de las aspiraciones vinculada a la generación de una ciudadanía que tenga precisamente como una de sus características la posibilidad de convivir en paz y en tolerancia con los otros. Convivir con los conocimientos es un despropósito. No se convive con los saberes, se les usa, se les pone en funcionamiento, se utilizan, se les cuestiona.

3. Al mantener como eje nucleador de la disciplina la meta de generar identidad entendiendo por ésta la construida entre los habitantes de un Estado nación específico- la intención de avanzar en la construcción de un pensamiento crítico expresa una contradicción irresoluble, pues éste tendría como límite para su ejercicio a la identidad nacional, la cual se asume como la única que debe ser enseñada. Esto se infiere por el uso del singular al hablar de "la identidad" que excluye en el discurso al resto de posibilidades identitarias, de adscripciones sociales, culturales, económicas y políticas que son borradas, desaparecidas, desdeñadas y ocultadas bajo el discurso de lo nacional. Incluso, para el caso mexicano, contraviene el propio artículo segundo constitucional.

Más adelante, cuando el documento aborda los propósitos de cada nivel educativo, se encuentra otro dato revelador de las concepciones tradicionales sobre la forma de entender el devenir de la humanidad. En el punto 2 se afirma: "Ubicar en el tiempo y en el espacio los principales procesos de la historia del México y el mundo". (SEP 2017b, 383) Esta frase expresa uno de los puntos más cuestionados cuando se discute sobre la necesidad de construir y enseñar una historia que enfrente las nociones colonialistas que sostienen que la historia de ciertas civilizaciones europeas son las que otorgan sentido al desarrollo de absolutamente toda la humanidad, por lo cual se le denomina con el calificativo de "mundial" o peor aún "universal".

La historia mundial es concebida desde un lugar específico. Las nociones que la cimientan, los ejes de referencia cronológicos, geográficos y culturales que la definen se refieren a la historia 
construida desde la mirada europea exclusivamente. Desde ahí se marca la línea que nos servirá de guía. Las historias de otras regiones serán vistas de manera accesoria a ese gran eje que es la historia europea y su herencia (colonial) en el resto del mundo (Rodríguez, 2008).

Un claro ejemplo de estas filiaciones políticos historiográficas es el fuerte acento que el programa de primer grado de educación secundaria incluye el estudio de las revoluciones burguesas que permitieron la consolidación del capitalismo. Destaca ahí la Unidad de Construcción de aprendizaje (UCA) ${ }^{2}$ titulada: "La Independencia de las Trece Colonias de Norteamérica", pues con ella la Secretaría de Educación Pública propone que durante varias semanas los niños y niñas de México se dediquen a trabajar con profundidad y a detalle el proceso de independencia de los Estados Unidos. La coartada historiográfica para tan profunda atención a ese proceso de independencia radica en la necesidad de revisar el ciclo de las revoluciones burguesas que explican el surgimiento de la sociedad moderna de índole capitalista y liberal. Sin embargo, la definición de ese tema es evidentemente de carácter ideológico y político pues, en estrictos términos historiográficos sería mucho más importante -aunque igualmente cuestionable didácticamenteestudiar con tal nivel de profundidad la revolución francesa o, mejor aún, alguna de las independencias latinoamericanas las cuales sirven para ubicar las líneas de desarrollo histórico compartidas con esa región del planeta. ${ }^{3}$

\section{La dimensión histórica}

La "Descripción de los organizadores curriculares" constituye la columna vertebral del cuerpo de contenidos que se trabajarán en los cursos de historia. Los cinco ejes que organizan el aprendizaje son:

1. Construcción del pensamiento histórico

2. Civilizaciones

3. Formación del mundo moderno

4. Formación de los Estados nacionales

5. Cambios sociales e instituciones contemporáneas.

Realizar el análisis detallado de la propuesta para los cinco grados rebasa por mucho los límites cuantitativos de los que disponemos en este artículo. Por ello nos limitaremos a señalar algunas de las características centrales planteadas por los 5 ejes organizadores curriculares definidos para todos los grados escolares en los que se impartirá la materia de historia (tres de

${ }^{2}$ La UCA es un trabajo de investigación cuyo proceso está pensado para realizarse en varias semanas. La selección del tema es opcional. Esto produce una paradoja curricular: por un lado, se ubican en las UCAs sugeridas por la SEP temas fundamentales de la historia para que sean estudiados a profundidad, al mismo tiempo que dichos temas son optativos. De esta manera, se presentan "la Independencia de las Trece colonias" y el "Holocausto" como temas que pueden ser trabajados con fuentes primarias y con mayor extensión temporal. Sin embargo, dichos temas son a su vez optativos, por lo que un profesor puede seleccionar no trabajar con el Holocausto. La paradoja queda así: los importante debe ser trabajado en una UCA pero a su vez no tiene carácter obligatorio.

${ }^{3}$ No está de más señalar que el tema de las revoluciones de independencia latinoamericanas no aparece en ninguno de los programas del nuevo modelo educativo 
primaria y dos de secundaria). Posteriormente comentaremos algunos ejemplos ilustrativos de los "aprendizajes esperados" y "las orientaciones didácticas" de los programas de secundaria ya que ahí aparecen algunas cuestiones emblemáticas de la concepción histórica, ideológica y política que inspiró todo el plan de estudios.

La siguiente tabla rescata el orden general de los temas históricos que se trabajan por grado escolar: ${ }^{4}$

Desarrollo cronológico en los programas de estudio de la asignatura de Historia 2017

\begin{tabular}{|c|c|c|}
\hline GRADO & INICIA EN & TERMINA EN \\
\hline $4^{\circ}$ PRIMARIA & $\begin{array}{l}\text { Orígenes de la agricultura y de la vida } \\
\text { aldeana (en México). }\end{array}$ & $\begin{array}{l}\text { Nueva España: diversidad cultural } \\
\text { y comunicación con el mundo. }\end{array}$ \\
\hline $5^{\circ}$ PRIMARIA & $\begin{array}{l}\text { El fin de la Nueva España y el desarrollo de } \\
\text { la guerra de independencia }\end{array}$ & $\begin{array}{l}\text { El arte y la cultura popular en la } \\
\text { construcción del siglo XX. }\end{array}$ \\
\hline $6^{\circ}$ PRIMARIA & $\begin{array}{l}\text { Las civilizaciones } \\
\text { (Mesopotamia, Egipto, China, India, } \\
\text { Mesoamérica y el área Andina). }\end{array}$ & La revolución inglesa \\
\hline $1^{\circ}$ SECUNDARIA & Revoluciones, burguesía y capitalismo & La globalización. \\
\hline $2^{\circ}$ SECUNDARIA & $\begin{array}{l}\text { Los indígenas en el México actual. } \\
\text { La civilización mesoamericana y otras } \\
\text { culturas del México antiguo. }\end{array}$ & $\begin{array}{l}\text { El patrimonio cultural y artístico } \\
\text { en la etapa virreinal }\end{array}$ \\
\hline
\end{tabular}

(Fuente. SEP, 2017b) Se dejó de lado la información que aparece en el primer organizador curricular ("Construcción del conocimiento histórico)" pues en él se abordan algunas problematizaciones sobre la manera en que las narrativas históricas se construyen, por lo que es hasta el segundo organizador curricular ("Civilizaciones") cuando se enuncian los temas históricos concretos contemplados en los programas los cuales constituyen el objeto de análisis de este artículo.

Los “organizadores curriculares" evidencian una forma tradicional de asumir el proceso de enseñanza aprendizaje de la historia en por lo menos los siguientes puntos.

1. La definición de los temas centrales de estudio pues nuevamente ellos aparecen delineados por:

a) una concepción de la historia de la humanidad de índole eurocéntrica la cual se hace pasar por historia mundial. Por ejemplo, en sexto de primaria después de un inicio alentador en el que se revisan civilizaciones originarias ubicadas en diversas partes del planeta, el embudo analítico perfila para identificar como fundamental el aprendizaje únicamente de la historia europea. Esa es la razón por la cual el programa de ese grado concluye con la revisión de la revolución inglesa, a la vez que el inmediato posterior ( $1^{\circ}$ secundaria) inicia con el ciclo de las revoluciones burguesas y el nacimiento del capitalismo, finalizando con el estudio de la globalización desde la perspectiva europea de dicho fenómeno.

${ }^{4}$ En síntesis y para mayor claridad de lector: historia nacional se trabaja en $4^{\circ}, 5^{\circ}$ de primaria y $2^{\circ}$ de secundaria; mientras que historia mundial en $6^{\circ}$ de primaria y $1^{\circ}$ de secundaria. 
b) la construcción de los Estados nación como elementos definidores del devenir mundial alrededor de cuyas explicaciones gira la atención de otros posibles objetos analíticos. La posibilidad de comprender el ejercicio de la política por fuera de esos rangos institucionales a fin de referirse a las múltiples expresiones cotidianas en la que los sujetos sociales ejercen o resisten al poder simplemente es inconcebible. Lo político para la historia escolar termina circunscribiéndose a la política referida ésta como el accionar del Estado nación (Mouffe, 2011).

c) la imposibilidad de superar (o tan siquiera cuestionar) la concepción lineal del tiempo y la manera de narrar el devenir de la historia escolar. Nos referimos a la reproducción de una propuesta didáctica -fruto de una concepción historiográfica (política, epistemológica y filosófica)que señala que la comprensión de los hechos sociales contemporáneos debe hacerse siempre remontándose en primer lugar a los acontecimientos más lejanos cronológicamente para de ahí acercarse paulatinamente al presente, entretejiendo en el camino las explicaciones sobre las razones de las actuales condiciones económicas, políticas y sociales.

Esa manera de abordar el proceso de construcción y comprensión de la historia (del pasado más lejano hasta llegar a la actualidad) se contrapone a la propuesta de que su enseñanza habría de tomar como centro referencial al presente, para que a partir de ahí se problematice el pasado y, por ende, se explique la forma en que desde nuestra contemporaneidad deben realizarse las expediciones hacia aquel, a fin de identificar y entretejer los diversos elementos que explican los temas elegidos. Esta forma distinta a la tradicional de plantear el abordaje de los temas históricos permitiría que el estudiante desde su aquí y ahora comprenda mejor la manera en que aquellos acontecimientos sucedidos en el pasado han influido en su vida actual.

Vemos pues que los planes y programas de estudio de historia, a pesar de algunas novedosas estrategias para tratar de afrontar y enriquecer la reflexión, terminan asumiendo la concepción hegemónica moderna y occidental de que el devenir es una línea progresiva que siempre debe leerse y explicarse de forma unidireccional, empezando por el pasado más lejano para llegar al presente $\mathrm{y}$, posiblemente, encaminarse hacia el futuro.

\section{La dimensión epistemológica}

Otro ejemplo claro de cómo los programas del NME reproducen la noción tradicional de los sentidos de identidad fundamentales es un tema con un título muy prometedor diseñado para segundo de primaria: "El conocimiento histórico en un país colonizado". (SEP, 2017b: 414) De forma optimista podríamos creer que en ese espacio se abordará la manera en que una sola voz narrativa de la historia se ha hecho hegemónica en función de razones de poder, pero que frente a ella existen muchas otras posibilidades -igual de legítimas y válidas- de contar, narrar, construir y escribir las múltiples historias existentes. Pero no es así, ya que los aprendizajes esperados para dicho tema son: a) reflexionar sobre la relación entre la historia nacional y la historia mundial, y, b) analizar las razones por las que la historia de occidente es también parte de nuestra historia. Es decir, se desarrollarán nociones que anhelan justificar las razones de aprender una única voz narrativa conformada por una visión occidental de la sociedad y por la historia nacional, por lo cual se comprende que no se haga referencia alguna a la existencia de otras historias, es decir, a la manera en que otras culturas explican su existencia. El tratamiento de la diversidad se queda, una vez más, tan solo a nivel de buenas intenciones declarativas. 
Por su parte el programa de tercer grado de secundaria abunda en ejemplos para el análisis. Revisemos solo algunos.

1. El penúltimo tema del eje "Formación de Estados nacionales", lleva por nombre "La identidad nacional". (SEP 2017b: 415) Sus aprendizajes esperados son una muestra de la total ausencia de crítica respecto al tema pues, recordemos, la razón de ser de enseñar historia es justamente la creación de dicha identidad. Así, el objetivo de los aprendizajes ahí planteados es argumentar sobre la necesidad histórica y presente de sostener a la identidad nacional como la única identidad posible, esperando que el alumno: a) reflexione sobre la relación entre diversidad nacional y unidad nacional, b) reconozca el papel histórico del nacionalismo, y, c) analice la vigencia y el sentido del nacionalismo hoy. En esos párrafos no hay una sola palabra sobre la existencia de otras identidades que todo sujeto social encarna y que son cubiertas, negadas, desdeñadas por la de índole nacional.

2. En el eje "Cambios sociales e instituciones contemporánea" hay dos temas claramente representativos de la posición política desde la cual se crearon estos programas de estudio. Uno es "El Estado y las instituciones" cuyos aprendizajes esperados parecieran haber sido rescatados de la plataforma propagandística del Partido Revolucionario Institucional (PRI), institución política que llevó al gobierno a los impulsores de la reforma educativa. Ellos son:

- "Analiza el proceso de estabilización política que se dio a partir de la fundación de un partido hegemónico y la consolidación de una presidencia fuerte.

- Reconoce el proceso de creación de instituciones e identifica los avances del Estado mexicano en educación y salud.

- Identifica las metas de la Reforma agraria y valora su impacto en las diferentes regiones." (SEP, 2017b, 416)

Los jóvenes que cursan tercer grado de secundaria recibirán información detallada acerca de las bondades y beneficios conseguidos con la instauración del sistema político surgido de la revolución de 1910 el cual refiere los grandes logros políticos, económicos y sociales del hasta hace apenas unos días (30 noviembre 2018) partido gobernante hegemónico por casi ocho décadas. (Cabe mencionar que en todo proceso electoral el PRI utiliza justamente como una de sus estrategias propagandísticas el señalamiento de que gracias a él en México existen instituciones y estabilidad social).

Finalmente, dentro de este eje el objetivo de aprender la importancia de la consolidación de una "presidencia fuerte" como elemento de estabilización, otorga un justificante y soporte ideológico a la existencia de un régimen presidencialista que en los hechos anuló la división de poderes otorgando un poder absoluto a una sola persona (los presidentes en turno).

En dos temas posteriores de nombre "Democracia, partidos y derechos políticos" y "Fortalecimiento de la democracia" (SEP, 2017b, 416) llama la atención que la línea argumentativa sobre el desarrollo de la democracia siempre es consecuente con la concepción de que ella se restringe a una concepción electoral, de sistema de partidos, representativa y procedimental. Tan es así que jamás se habla de la existencia de otras posibles formas de ejercicio democrático (directa, popular, etc.), y sí en cambio se enfatiza la reforma electoral que se generó en la década de los setenta como consecuencia de los acontecimientos de 1968 y el rol que a partir de la década de los noventa ha tenido el Instituto Nacional Electoral (INE) en la consolidación de esa democracia en México. 
De esta manera, la dimensión epistemológica del programa de historia para la educación obligatoria en México presenta y reproduce una sola forma de comprender la historia, una sola forma de identidad nacional y un solo sujeto de la historia. El resultado es, evidentemente, la reproducción del viejo código disciplinar de la historia que sólo permite pensar la historia desde occidente, excluyendo la pluralidad identitaria y únicamente narra la historia del poder representado en el Estado-nación.

\section{Conclusiones}

México se encuentra en un momento convulso de su historia y su sistema educativo no está exento a estos grandes cambios. La reforma educativa de 2012 con respecto a las condiciones laborales de los docentes y de 2017 con respecto a los contendidos curriculares para la educación básica son la conclusión histórica de un largo proceso histórico de reforma educativa iniciado en los años setenta del siglo XX y desarrollado con fuerza a partir de la última década del siglo pasado. Tras el reciente triunfo electoral de Andrés Manuel López Obrador en julio de 2018, dicho proceso parece estar llegando a su fin.

El gobierno de López Obrador (2018-2024) ya ha mostrado indicios de un interés particular en la enseñanza de la historia nacional. Sin embargo, su gusto por los grandes héroes y su mirada Estado céntrica muestra más una reproducción del código disciplinar de la historia (Cuesta, 2018) que visos de una historia más inclusiva y plural. Por esto, la construcción de una historia escolar diferente debe asumir la diversidad cultural, la cotidianidad del ejercicio político, una visión decolonizada de temas, periodizaciones, formas de acercamiento que abran nuevos cuestionamientos en las aulas y los diseños curriculares que faciliten el desarrollo de una pedagogía por la justicia social.

De no llevarse a cabo loa anterior, como hemos demostrado en nuestro análisis de la reforma curricular de 2017 y recuperando el texto clásico de Giuseppe Tomasi de Lampedusa, estamos en posibilidad de afirmar que en los planes y programas de historia surgidos de la reforma educativa todo se cambió para que todo permaneciera igual. Este gatopardismo pedagógico puede seguir reproduciéndose en el futuro, especialmente una visión lineal, eurocéntrica, homogénea y Estado céntrica de la historia dentro de las escuelas.

\section{Referencias bibliográficas}

Abellán Fernández, J. (2018). El programa de Historia para la educación básica 2018 en México. Concepciones sobre el aprendizaje, las estrategias y las actividades didácticas. Clío y Asociados, No. 26, pp. 38-57

Aboites, H. (2012), La medida de una nación: los primeros años de la evaluación en México. Historia de poder y resistencia (1982-2012). México, Clacso/uam/Ítaca.

Alcántara, A. (2008), Políticas educativas y neoliberalismo en México: 1982-2006. Revista Iberoamericana de Educación. núm. 48, pp. 147-165.

Apple, M. (2012). Poder, conocimiento y reforma educacional. Buenos Aires, Miño y Dávila Editores. 
Arnaut, A. (1998). Historia de una profesión: Los maestros de educación primaria en México, 1887-1994. México: Sep.

Ballesteros Páez, M. (2015). Race and racism in Mexican history textbooks:A silent presence. En M. Araújo, L. Maseo, et.al., Eurocentrism, Racism and Knowledge (pp. 209-228). Hampshire: Palgrave Macmillan.

Ballesteros, M. D. (2012) Cambios y continuidades en los contenidos curriculares de los programas de historia de México de educación secundaria (1993-2011). Memoria del Tercer Encuentro Nacional de Docencia, Difusión y Enseñanza de la Historia y Primer Encuentro Internacional de Enseñanza de la Historia (pp. 17-32). México: Universidad Pedagógica Nacional

Casal, Silvana (2011). Aprender historia en la escuela secundaria. El caso de Morelia, Michoacán (México), Revista Mexicana de Investigación Educativa (México), vol. 16, núm. 48

Chapman, Th. y Hobbel, N. (2010). Introduction. Social Justice Pedagogy Across the Curriculum. The Practice of Freedom, Nueva York, Routledge.

Chervel, A. (1991). Historia de las disciplinas escolares. Reflexiones sobre un campo de investigación, Revista de Educación, 295, pp. 59-111.

Chevallard, Y., (1997). La transposición didáctica. Del Saber Sabio al Saber Enseñado. Buenos Aires: AIQUE.

Coordinadora Nacional de Trabajadores de la Educación (CNTE) (2013). Posición de la CNTE ante la contrarreforma educativa expuesta en los foros Análisis y perspectivas de la reforma educativa. Análisis y perspectivas de la reforma educativa. Memorias y resolutivos, México, cnte, julio de 2013, en 〈http://www.rebelion.org/docs/171157. pdf〉, consultado el 23 de mayo de febrero de 2018.

Cuesta, R. (1998). Clío en las aulas. La enseñanza de la historia en España entre reformas, ilusiones y rutinas. Madrid: Akal.

Diaz Barriga, A. (2016). Propuestas de Ángel Díaz Barriga para modificar la reforma de la SEP. Insurgencia magisterial. http://insurgenciamagisterial.com/propuestas-de-angel-diazbarriga-para-modificar-la-reforma-de-la-sep/. Consultado 21 de mayo de 2018.

García Garduño, J. (2014). Estudio introductorio. Pinar, W. La teoría del curriculum. Madrid: Narcea, 11-60.

Gil Antón, M. (2013) La reforma educativa. Colmex. https://www.youtube.com/watch?v=jH6lzKYGgNY, Consultado 22 de mayo de 2018.

González Arroyo, M. (2015). El contenido en las escuelas de los pobres. José Gimeno Sacristán (comp.), Los contenidos. Una reflexión necesaria. Madrid: Morata. 
Goodson, I. F. (1995). Historia del curriculum. La construcción social de las disciplinas escolares. Barcelona: Pomares-Corredor.

H. Dumont, D. Istance y F. Benavides (2010), The Nature of Learning: Using Research to Inspire Practice, Paris: OCDE.

Latapí, Pablo. (1998) "Un siglo de educación nacional: una sistematización”, en Pablo Latapí (coord.) Un siglo de educación en México I . México, Fondo de Cultura Económica, 1998, pp. 21-42.

Legardez, A. (2003). L'Enseignement des Questions sociales et historiques, socialement vives. Le cartable de Clio, 3 .

Meyer, Lorenzo (1995), Liberalismo autoritario. Las contradicciones del sistema político mexicano, México, Océano.

Mignolo, W. (2003). Historias locales/diseños globales: colonialidad, conocimientos subalternos y pensamiento fronterizo. Madrid: Akal.

Mouffe, C., (2011). En torno a lo político, México: Fondo de Cultura Económica.

OCDE, Centre for Educational Research and Innovation (2012). La Naturaleza del Aprendizaje. Investigación para inspirar la práctica, ¿Cómo pueden las ciencias del aprendizaje aportar al diseño de los ambientes de aprendizaje del siglo XXI?, Disponible en: $<$ http://www.oecd.org/education/ceri/The\%20Nature\%20of\%20Learning.Practitioner\%20 Guide.ESP.pdf $>$ Consultado el 25 de junio de 2018.

Ornelas, Carlos (2000). El sistema educativo mexicano. La transición de fin de siglo, México, fce.

Ornelas, Carlos (2012). Educación, colonización y rebeldía: la herencia del pacto CalderónGordillo. México, Siglo XXI Editores.

Pacheco Ladrón de Guevara, L.; Navarro Hernández, M. y Cayeros López, L. (2011). Los pueblos indios en los libros de texto gratuitos. Revista Mexicana de Investigación Educativa, 49, 525-544.

Pérez Expósito (2014) Rethinking political participation: A pedagogical approach for citizenship education. Theory and Research in Education 1-23

Pinar, W. (2004). What is Currículum Theory? Mahwah, N. J.: Lawrence Erlbaum.

Plá, S. (2014) Ciudadanía y competitividad en la enseñanza de la historia. Los casos de México, Argentina y Uruguay, México, Universidad Iberoamericana, Departamento de Historia

Plá, S. (2014a) "Mestizos e inconclusos. Interpretaciones sobre la Historia de México en bachillerato", Revista Mexicana de Investigación Educativa, México, COMIE, vol. 19, no. 61, abril-junio 
Plá, S. (2016). Currículo, historia y justicia social. Estudio comparativo en América Latina, Revista Colombiana de Educación, núm. 71, julio-diciembre, 2016, pp. 53-77 Universidad Pedagógica Nacional Bogotá, Colombia. Recuperado de: http://www.scielo.org.co/pdf/rcde/n71/n71a03.pdf

Plá, S. (2012). La enseñanza de la historia como objeto de investigación. Secuencia [163] núm. 84, septiembre-diciembre.

Quijano, A. (2000). Colonialidad del poder, eurocentrismo y América Latina. En E. Lander (comp.). La colonialidad del saber: eurocentrismo y ciencias sociales. Perspectivas Latinoamericanas. Buenos Aires: Clacso.

Rockwell, E. (2015). 20 preguntas iniciales. Nexos, https://educacion.nexos.com.mx/?p=5, consultado 21 de mayo de 2018.

Rodríguez, X. (2008). Una historia desde y para la interculturalidad. México: Universidad Pedagógica Nacional.

Rodríguez, X. y Velasco, S. (2014). Otredad y construcción de futuro. La educación para los indígenas en México, un balance histórico. Revista Española de Educación Comparada, 23.

Santisteban, A. (2010). La formación de competencias de pensamiento histórico. Clío \& Asociados, 14, 34-56. Recuperado de: http://www.memoria.fahce.unlp.edu.ar/art_revistas/pr.4019/pr.4019.pdf

Secretaria de Educación Pública (SEP) (2017a). Nuevo Modelo Educativo para la educación obligatoria. Educar para la libertad y la creatividad. México: SEP. Recuperado de: < https://www.gob.mx/cms/uploads/attachment/file/198738/Modelo_Educativo_para_la_Ed ucacio_n_Obligatoria.pdf>

Secretaria de Educación Pública (SEP) (2017b). Aprendizajes clave para la educación integral. Plan y programas de estudio para la educación básica, México: SEP.

Tedesco J. C. (2016). Tendencias actuales de las reformas educativas, Revista Estudios, [S.1.], n. 5, p. 85-92, mar. 2016. ISSN 1852-1568. Recuperado de: $\langle$ https://revistas.unc.edu.ar/index.php/restudios/article/view/14116/14333〉. Fecha de acceso: 01 Jul. 2018

Viñao, A. (2006). La historia de las disciplinas escolares. Historia de la educación, 25, Ediciones Universidad De Salamanca Hist. educ., 25, pp. 243-269

Walsh, C., (2006). Interculturalidad y colonialidad del poder: Un pensamiento y posicionamiento otro desde la diferencia colonial. Walsh C., García A., Mignolo, W. Interculturalidad, descolonización del Estado y del conocimiento. Buenos Aires: Signo.

Young, K. (2010). Progreso, patria y héroes. Una crítica del currículo de historia en México. Revista Mexicana de Investigación Educativa, 45, 599-620. 